\title{
M018
}

\section{EXPERIMENTAL ASSESSMENT OF THE ACCURACY OF GENOMIC SELECTION IN SUGARCANE}

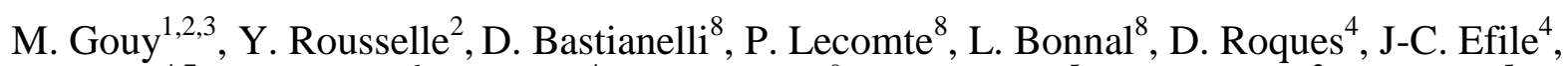

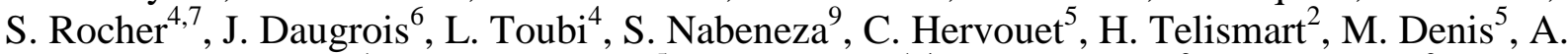 \\ Thong Chane ${ }^{1}$, J.C. Glaszmann ${ }^{5}$, J.-Y. Hoarau ${ }^{1,4} *$, S. Nibouche ${ }^{2}$ and L. Costet ${ }^{2}$ \\ ${ }^{1}$ eRcane, F-97494 Sainte-Clotilde, La Réunion, France \\ ${ }^{2}$ Cirad, UMR PVBMT, F-97410 Saint-Pierre, La Réunion, France \\ ${ }^{3}$ Université de la Réunion, UMR PVBMT, F-97410 Saint-Pierre, La Réunion, France \\ ${ }^{4}$ Cirad, UMR AGAP, F-97170 Petit Bourg, Guadeloupe, France \\ ${ }^{5}$ Cirad, UMR AGAP, F-34398 Montpellier, France \\ ${ }^{6}$ Cirad, UMR BGPI, F-97170 Petit Bourg, Guadeloupe, France \\ ${ }^{7}$ Université des Antilles et de la Guyane, F-97157 Pointe-à-Pitre, Guadeloupe, France \\ ${ }^{8}$ Cirad, UMR SELMET, F-34398 Montpellier, France \\ ${ }^{9}$ Cirad, UMR SELMET, F-97410 Saint-Pierre, La Réunion, France \\ *E-mail: jean-yves.hoarau@cirad.fr
}

Key words: Saccharum spp., genomic selection, morphological traits, technological traits, disease resistance, statistical models.

Sugarcane cultivars are interspecific hybrids with an aneuploid, highly heterozygous polyploid genome. The complexity of the sugarcane genome is the main obstacle to the use of marker assisted selection in sugarcane breeding. Given the promising results of recent studies of plant genomic selection, we explored the feasibility of genomic selection in this complex polyploid crop. Genetic values were predicted in two independent panels, each composed of 167 accessions representing sugarcane genetic diversity worldwide. Accessions were genotyped with 1499 DArT markers. One panel was phenotyped in Reunion Island and the other in Guadeloupe. Ten traits concerning sugar and bagasse contents, digestibility and composition of the bagasse, plant morphology and disease resistance were used. We used four statistical predictive models: bayesian LASSO, ridge regression, reproducing kernel Hilbert space and partial least square regression. The accuracy of the predictions was assessed through the correlation between observed and predicted genetic values by cross-validation within each panel and between the two panels. We observed equivalent accuracy among the four predictive models for a given trait, and marked differences were observed among traits. Depending on the trait concerned, within-panel cross validation yielded median correlations ranging from 0.29 to 0.62 in the Reunion Island panel and from 0.11 to 0.5 in the Guadeloupe panel. Cross validation between panels yielded correlations ranging from 0.13 for smut resistance to 0.55 for brix. This level of correlations is promising for future implementations. Our results provide the first validation of genomic selection in sugarcane.

Published in Gouy et al. 2013. TAG, 126:2575-2586. http://dx.doi.org/10.1007/s00122-013-2156-z 